ISSN 2504-1916 VOL. 16/1 (2017), 208-220

\title{
Die DDR und der Jazz in den 1960er-Jahren
}

\section{Aufbau von Jazzclubs und Konzertreihen hinter der Berliner Mauer}

\author{
Katharina Weißenbacher
}

\section{Einleitung}

$\mathrm{I}$ der DDR entwickelte sich nach dem Mauerbau eine spezifische Weise, Jazz zu interpretieren und sich frei zu spielen. Nachdem sich die Jazzmusiker über mehrere Jahre hinweg epigonal an ihren amerikanischen Vorbildern orientiert hatten, gelang es ihnen, sich im Laufe der 1960er-Jahre von ihren Idolen zu lösen und ihre eigene Musik zu formen. In einem Land, das durch eine Mauer geteilt war, wurde ihre Musik so zum Freiheitssymbol.

Die 1960er-Jahre galten im Hinblick auf die Entwicklung des Jazz in der DDR als eine Zeit des Wandels. Jazz galt anfangs als dekadent, da die amerikanische Unterhaltungsindustrie als kapitalistisch angesehen wurde und sich diese Ansätze und die Idee des ,American Way of Life، nicht mit der ,DDR-Ideologie ${ }^{6}$ des Marxismus-Leninismus vereinbaren ließen. Das DDR-Regime war bestrebt, die Kulturpolitik in der DDR nach dem sowjetischen Vorbild neu zu gestalten, stand aber gleichzeitig unter Druck, das Image der DDR, das durch den Mauerbau von 1961 gelitten hatte, aufzupolieren und die Unzufriedenheit des Volkes zu dämpfen. Um die Bevölkerung in gewissem Sinne ,aufzuheitern', bediente sich das Regime der Unterhaltungsmusik. Jazz erreichte in der DDR erst nach und nach Akzeptanz durch das Regime und damit verbundene Förderung. Die Situation blieb jedoch weiterhin ambivalent: Nachdem das Regime den Jazz durch die immer größer werdende Popularität der Beatmusik Mitte der 1960er-Jahre aus dem Fokus verloren hatte, fanden die Funktionäre Mitte der 1970er-Jahre selbst Gefallen am Jazz, da sich dieser - insbesondere der Free Jazz - dazu instrumentalisieren ließ, im Westen ein liberales Image der DDR zu vermitteln. Der anfangs verpönte Jazz in der DDR wurde so zum Kulturexport. Welches waren nun die entscheidenden Stationen auf diesem dialektischen Weg?

\section{Jazz in der DDR}

„Zwar benötigte man in der DDR für so ziemlich alles eine Genehmigung, aber die Musiker konnten sich in ihrer Musik frei ausdrücken“"1; so äußerte sich der Pianist Joachim Kühn in der

1 Joachim Kühn im Film „Play your own thing - Eine Geschichte des europäischen Jazz“, Julian 
Rückschau. Die Musiker in der DDR mussten sich mit diversen bürokratischen Rahmenbedingungen wie der 60:40-Regelung ${ }^{2}$ arrangieren, konnten aber in ihrer Musik frei improvisieren und durch musikalische Mittel ihren Gefühlen Ausdruck verleihen. Musiker in der DDR benötigten dabei eine Spielerlaubnis, die sie nur durch ein Studium an einer Musikhochschule oder durch eine Berufsausbildung an der Musikschule Berlin-Friedrichshain erlangen konnten. ${ }^{3}$ Kultur wurde in der DDR vom Staat finanziert, jedoch war es zu Beginn der 1960er-Jahre kaum möglich, allein mit Jazz eine Existenz zu sichern, da im Bereich der Populärmusik lediglich Tanz- und Unterhaltungsmusik gefördert wurde. Viele Jazzmusiker in der DDR begannen ihre künstlerische Laufbahn in diversen Tanzorchestern wie dem Orchester Eberhard Weise und dem Rundfunk-Tanzorchester Berlin; erst ab den frühen 1970er-Jahren gelang es den Musikern, ihren Lebensunterhalt ausschließlich oder immerhin mehrheitlich durch ihre Jazzaktivitäten zu bestreiten. ${ }^{4}$ Nur einzelne schafften dies bereits in den 1960er-Jahren, wie beispielsweise der Pianist Joachim Kühn. Mit seinem Joachim Kühn Trio ${ }^{5}$ wurde er ab 1965 u. a. im Rathauskeller in Potsdam für regelmäßige Auftritte engagiert und stand häufig mit seinem Bruder, dem Klarinettisten Rolf Kühn, der sich schon vor dem Bau der Mauer in den Westen abgesetzt hatte, auf der Bühne. ${ }^{6}$ Im Jahre 1966 verließ auch Joachim Kühn die DDR über Österreich, da er sich in der DDR in der Ausübung seiner Musik nicht frei genug fühlte. ${ }^{7}$ Er beschrieb die Situation des Jazz in der DDR in den 1960er-Jahren folgendermaßen:

„Jazz war in der DDR eigentlich unmöglich. Jazzmusiker gab es da eigentlich gar nicht. Da gab es nur Tanzmusiker, die als Hobby Jazz spielten. Allen voran Ernst-Ludwig Petrowsky und eine TanzBand, in der auch ich spielte - das Werner Pfüller-Quintett. Die wollten zwar gerne Jazz spielen, aber es gab keine Möglichkeit, sich damit seinen Lebensunterhalt zu verdienen. Das gab's nicht. Und es gab in der DDR so Leute - DDR-Bonzen haben wir die genannt - die in den Jugendklub kamen und uns erzählten, dass Jazz amerikanische imperialistische Musik ist und dass es für die DDR nicht gut ist, solche Musik zu spielen. Dann mussten wir die aufklären, dass der Ursprung des Jazz eigentlich bei der Sklavenmusik ist und das Ganze mit Imperialismus nichts zu tun hat, sondern Musik ist, die von einem Volk kommt. Dann war es mal wieder erlaubt, dann war es verboten.

Benedikt, DVD 2007 [keine Bestellnummer vermerkt]. Delphi Filmverleih GmbH, Kurfürstendamm 226, 10719 Berlin.

2 Diese Regelung, die besagte, dass jede Band aus mindestens $60 \%$ einheimischen Musikern bestehen musste, war Bestandteil der Anordnung über die Programmgestaltung bei Tanz- und Unterhaltungsmusik vom 2. Januar 1958 des Ministeriums für Kultur der DDR

3 Grundlage für diese Spielerlaubnis bildete die Anordnung über die Befugnis zur Ausübung von Tan₹- und Unterhaltungsmusik aus dem Jahre 1953, die anfangs nur für Berufsmusiker festlegt worden war. Erst im Jahre 1957 wurde diese für Laienmusiker erweitert.

4 Ekkehard Jost, Europas Jazz 1960-1980, Frankfurt am Main: Fischer 1987, S. 235.

5 Mit Klaus Koch am Bass und Reinhard Schwartz am Schlagzeug.

6 Musiker aus der DDR durften in ihrer Heimat nie gemeinsam mit Musikern aus der BRD auftreten, es sei denn, sie hatten einen sogenannten „Alibi-Ausländer“ in der Band. Die Brüder Rolf (cl) und Joachim Kühn (p) spielten beispielsweise Anfang der 1960er-Jahre gemeinsam mit den beiden polnischen Musikern Michael Urbaniak (sax) und Czeslaw Bartkowski (dr). Rolf Kühn lebte zu dieser Zeit bereits im Westen, Joachim Kühn noch bis 1966 in der DDR.

7 Joachim Kühn, persönliches Interview, telefonisch geführt von Katharina Weißenbacher, 27.1.2014. 
Es gab keine richtige Jazzsendung im Radio, außer von Karl-Heinz Drechsel, und Platten gab es sowieso nicht. Also ich hatte immer mehrere Platten, alleine schon von meinem Bruder ${ }^{8}$, der schon immer in Westdeutschland war, und ich war auch die ganze Nacht am Radio und habe jede Jazzsendung mitgeschnitten. Ich war richtig enthusiastisch.“9

Joachim Kühns Beschreibung „dann war es mal wieder erlaubt, dann war es verboten“ zeigt das ambivalente Verhältnis des politischen Systems der DDR zum Jazz auf. Anfangs wurde der Jazz als dekadente und sogar als ,Affen-Musik' betitelt und erregte als Musik, die ihren Ursprung in Amerika hatte, bei den Funktionären besonderen Argwohn. ${ }^{10}$ Doch im Laufe der Jahre erkannte das Regime die Möglichkeit, Jazzmusiker für eigene Zwecke zu instrumentalisieren. Jazzmusiker wurden zu Devisenbringern und zum ,Kulturexportartikel'; besonders Free-Jazzer wurden gerne für Konzerte in den Westen entsandt, um dort ein liberales Image der DDR zu repräsentieren. ${ }^{11}$

In den 1960er-Jahren gab es zahlreiche ideologische Richtungswechsel im Bereich der Kulturpolitik $^{12}$ von Seiten der SED, und dies betraf auch Jazzmusiker, Schriftsteller und weitere Künstler. Nachdem der Versuch, die Kulturpolitik beispielsweise durch den Bitterfelder Weg ${ }^{13}$ neu auszurichten, nicht den erwünschten Erfolg gebracht hatte, kam es im Jahre 1965 beim 11. Plenum des ZK der SED zu einem ,Kahlschlag“ im Bereich der Kulturpolitik: zahlreiche DEFAFilme wurden zensiert und mehrere Auftrittsverbote ausgesprochen. Diese Verbote trafen u. a. die Jazzsängerin Ruth Hohmann und den Liedermacher Wolf Biermann, der im Jahre 1976 aus der DDR ausgewiesen wurde. ${ }^{14}$

Nach Joachim Kühns Ausreise im Jahre $1966^{15}$ durften seine bis zu diesem Zeitpunkt produzierten Aufnahmen in der DDR nicht mehr gespielt oder veröffentlicht werden. Dazu

8 Rolf Kühn (cl).

9 Aus dem Telefoninterview mit Joachim Kühn vom 27.1. 2014.

10 Rainer Bratfisch, „Die fünfziger Jahre: Heißer Jazz im Kalten Krieg“, in: Freie Töne. Die Jazzszene in der DDR, hrsg. von dems., Berlin: Ch. Links Verlag 2005, S. 31-55, hier S. 44.

11 Ewald König, Warum Honecker zum Jaz:fan wurde. www.euractiv.de/europa-2020-und-reformen/artikel/ warum-honecker-zum-jazzfan-wurde-003892, eingesehen am 26.7.2015.

12 Um der Unzufriedenheit im Volk entgegenzuwirken, gab es im Jahre 1963 einige Lockerungen im Bereich der Kulturpolitik. Siehe Abschnitt „Lyrik - Jazz - Prosa“.

13 Der Bitterfelder Weg verfolgte ab 1959 das Bestreben, die Arbeiter mit einem aktiven Zugang der Kunst und Literatur näherzubringen. Eine neue programmatische Entwicklung der sozialistischen Kulturpolitik war geplant, und mit dem Motto Greif zur Feder, Kumpel, die sozialistische deutsche Nationalkultur braucht dich! versuchte das politische Regime diese durchzusetzen. Vgl. Gunther Ruther, Greif zur Feder Kumpel. Schriftsteller, Literatur und Politik in der DDR 1949-1990, Düsseldorf: Droste 1992.

14 Vgl. www.erinnerungsort.de/_- 501.html, eingesehen am 2.5.2017. Zu Aufführungsverboten und Ausbürgerung siehe auch Günter Agde (Hrsg.), Kablschlag. Das 11. Plenum des ZK der SED 1965. Studien und Dokumente, Berlin: Aufbau-Taschenbuch-Verlag 1991; Bratfisch, Freie Töne; Dietmar Keller und Matthias Kirchner (Hrsg.), Biermann und kein Ende. Eine Dokumentation zur DDR-Kulturpolitik, Berlin: Dietz 1991; Fritz Pleitgen (Hrsg.), Die Ausbürgerung. Anfang vom Ende der DDR. Wolf Biermann und andere Autoren, Berlin: Ullstein 2006.

15 Joachim Kühn nahm an einem Wettbewerb für junge Pianisten in Österreich teil, dessen Organisator der österreichische Pianist und Komponist Friedrich Gulda (1930-2000) war. Vor dem Abschluss- 
zählte u. a. die Platte Solarius, die 1964 mit seinem Bruder Rolf Kühn an der Klarinette, Klaus Koch am Bass und den beiden polnischen Musikern Michael Urbaniak (sax) und Czeslaw Bartkowsky (dr) bei der staatlichen Plattenfirma AMIGA entstanden war. Joachim Kühn bewegte sich als einer der ersten Jazzmusiker in der DDR in Richtung freies Spiel, indem er sich von rhythmischen und tonalen Vorgaben löste; aber auch andere Musiker waren in der Entwicklung ihrer eigenen Musik einfallsreich. Beispielsweise schrieb der Jazzpianist Ulrich Gumpert im Jahre 1972 für seine Ulrich Gumpert Workshop Band eine Suite aus teutschen Landen und bezog sich hierbei auf die Rezeption von deutschen Volksliedern. Die Melodien der ausgewählten Stücke wurden als Grundlage für die darauffolgenden Improvisationen verwendet und somit zum Thema der einzelnen Titel der Suite. Gumpert notierte jeweils das Thema und ließ die Musiker darüber frei improvisieren. Die Musiker bewegten sich in ihrer Improvisation vom Thema weg und kehrten im Laufe des Titels oder zu Ende der Improvisation zu diesem zurück. ${ }^{16}$ Dabei fungierten diese Themen der Volksmusik nicht als Zitate, sondern sie wurden durch Stilmittel des Free Jazz verfremdet und in den musikalischen Kontext integriert. ${ }^{17}$

Schon 1961 tauchte die Idee, über Volkslieder zu improvisieren, in einem Bericht des Ministeriums für Kultur der DDR über den hiesigen Jazz auf. Dieser Bericht wurde am 15. Dezember 1962 in einer Beratung über den Jazz verfasst:

„Die Musiker berufen sich in ihren Begründungen, warum sie Jazz spielen, oft auf die darin enthaltenen Möglichkeiten der Improvisation. Gegen das Improvisieren in der Musik ist nichts einzuwenden. Dieses belebende Mittel sollte aber benutzt werden, um zu erreichen, dass die Musiker nicht nur über amerikanische Jazzmusik, sondern auch über Themen unserer Tanzmusik, über Themen aus der Folklore anderer Völker, insbesondere der des sozialistischen Lagers und der im Befreiungskampf stehenden Völker improvisieren." ${ }^{18}$

Auch die Gruppe Synopsis, die im Jahre 1973 gegründet wurde, spielte Jahre später Gumperts Suite aus teutschen Landen für das Schweizer Label Intakt Records ein. Mit Ulrich Gumpert am Klavier, Conny Bauer an der Posaune, Ernst-Ludwig Petrowsky am Saxophon und Günter „Baby“ Sommer am Schlagzeug benannte sich Synopsis im Jahre 1984 in Anspielung auf das Zentralkomitee in Zentralquartett um. Um keine Gelegenheit zur Provokation verlegen, stellten die vier Musiker für das Schallplattencover von Zentralquartett das berühmte Bild mit den Drei-

konzert hatte sich Joachim Kühn bereits abgesetzt und es gelang ihm durch die Hilfe seines Bruders Rolf, im Westen musikalisch schnell Fuß zu fassen.

16 Vgl. Ulrich Gumpert, Suite nach Motiven deutscher Volkslieder. Jazz-Werkstatt-Orchester: Jochen Gleichmann (tp), Klaus Richter (tp), Conny Bauer (tb), Sieghardt Schubert (tb), Ernst-Ludwig Petrowsky (ss), Manfred Hering (as), Helmut Forsthoff (ts), Manfred Schulze (bars), Ulrich Gumpert (e-p, ld), Gerd Lübke (b), Christoph Niemann (b), Günter Sommer (dr), Wolfgang Winkler (dr), LP AMIGA 855549 (1977), CD AMIGA 0208059CTT (2012) (aufgenommen am 4.9.1972, Berlin „Jazz in der Kammer" Nr. 48).

17 Jost, Europas Jaz:, S. 248.

18 Beratung über Jazz am 15.12.1961 beim Ministerium für Kultur. Stiftung Archiv der Parteien und Massenorganisationen der DDR im Bundesarchiv Berlin (SAPMO-BArch), DY 24/6729. 


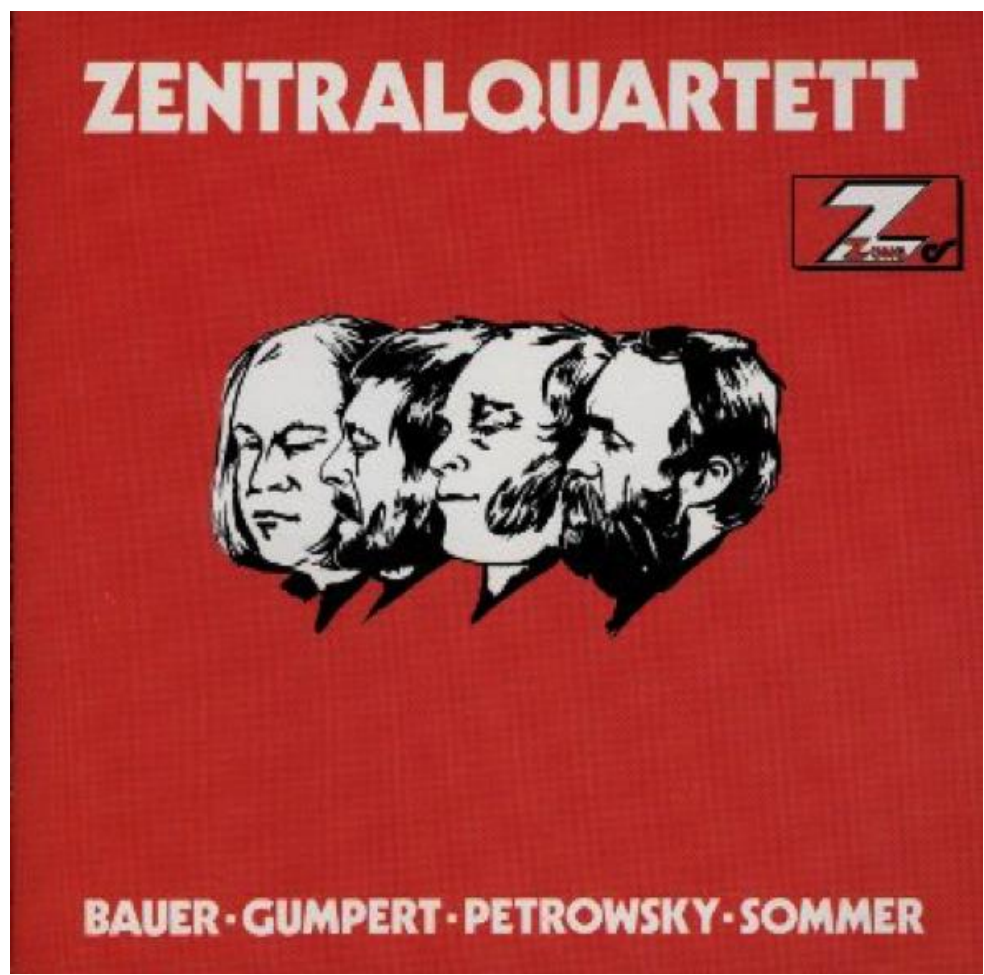

Abbildung 1: Das beschriebene Plattencover zu Zentralquartett, Idee und Gestaltung: Fritz Magull, Zeichnung: Sigurd Wendland ${ }^{19}$

viertelprofilen von Marx, Engels, Lenin und Stalin nach, und Ernst-Ludwig Petrowsky gab 1978 bei einem Auftritt in Hamburg (BRD) ein Stück mit dem Titel Ein Sandsack zu viel zum Besten, welches auf den ständigen Begleiter des Staatssicherheitsdienstes anspielte.

Im Jahre 1973 reiste die Gruppe Synopsis erstmals zum internationalen Jazzfestival Jazz Jamboree nach Warschau und präsentierte dort ihr freies Spiel. Ein Jahr später trat der westdeutsche Saxophonist Peter Brötzmann bei der Jazz Jamboree auf die Bühne und spielte seine eigene Version des Einheitsfrontliedes von Hanns Eisler gemeinsam mit Alexander von Schlippenbach (p), Paul Lovens (dr) und Peter Kowald (b): ein Auftritt, der die Musiker der DDR begeisterte und dazu führte, dass bald auch weitere DDR-Jazzer Themen aus Werken von Hanns Eisler in ihre Improvisationen aufnahmen. So spielten Gumpert und seine Kollegen bald einen „Eisler-Weill-Folklore-Freejazz“. ${ }^{20}$

Der Einfluss amerikanischer Jazzmusiker auch nach dem Mauerbau, ob im Radio oder durch Schallplatten die in die DDR geschmuggelt worden waren, lässt sich kaum überhören, wenn man die Musik der 1960er- und 1970er-Jahre etwas genauer studiert. So erinnert der

19 Mit freundlicher Genehmigung durch Sigurd Wendland.

20 Patrik Landolt, „Günter „Baby Sommer. Hör- und Sehmusik‘, in: Die lachenden Außenseiter. Musikerinnen und Musiker zwischen Jaz:, Rock. und Neuer Musik. Die 80er und 90er Jabre, hrsg. von Patrik Landolt und Ruedi Wyss, Zürich: Rotbuchverlag 1993, S. 129-137, hier S. 133. 
Groove des Beat Blues von Ernst-Ludwig Petrowsky ${ }^{21}$ an Herbie Hancocks Water Melon Man ${ }^{22}$, das Werner Pfüller-Quintett orientierte sich am Hard Bop und spielte Bobby Timmons Moanin' ein. Gemäß Bert Noglik brachte diese Aufnahme des Werner Pfüller-Quintetts die Entwicklung Richtung Hard Bop fast programmatisch zum Ausdruck. ${ }^{23}$ Begriff und Praxis des „Free Jazz“ wurden nicht in der DDR ,erfunden', sondern sie entwickelten sich auch hier nach dem Vorbild von Ornette Colemans Platte Free Jaz:. A collective improvisation, die im Jahre 1962 erschienen war.

Was den Jazz in der DDR ausmachte, setzte sich aus vielen Komponenten zusammen, seien es die freie Improvisation über ein Thema, zu dem man wieder zurückkehrt, die Publikumsnähe, das Aufbegehren in der Musik oder der ,Ersatz' für nicht ausreichend im Land präsente amerikanische Musiker. Auf jeden Fall haben sich hier bedeutende Musiker entwickelt, was sich auch darin bestätigt, dass diese nach dem Zusammenbruch der Berliner Mauer 1989 und der Deutschen Demokratischen Republik als Staat insgesamt schon im Jahr darauf im wiedervereinigten Deutschland als Jazzer wieder Fuß fassen konnten und auch heute noch in ausverkauften Sälen auftreten.

\section{Der Jazz und die FDJ - IG Jazz und erste Jazzclubs in der DDR}

Das Pendant zu den Hot Clubs in Westdeutschland bildeten in der DDR die sogenannten Interessengruppen Jazz, kurz IG Jazz. Bei Treffen der Interessengruppen hielten Jazzfans Vorträge über Jazz und hörten gemeinsam Schallplatten, organisierten aber auch Konzerte. Bereits in den 1950er-Jahren wurden in der DDR die ersten IG Jazz gegründet: beispielsweise im Januar 1955 in Leipzig (unter der Leitung von Reginald Rudorf) und in Halle, wo die ,arbeitsgemeinschaft jazz halle in der FDJ-Organisation der Martin-Luther-Universität" unter der Leitung von Alfons Zschockelt und Siegfried Schmidt-Joos gegründet wurde. Später schloss sich der Chemiestudent Günter Herbsleb an und die Leitung wurde zu einer Art Triumvirat. ${ }^{24}$ Im Jahr darauf, am 29. März 1956, von Karlheinz Drechsel in Dresden, im Dezember 1956 in Chemnitz (damals KarlMarx-Stadt) und in demselben Jahr eine IG Jazz Berlin. ${ }^{25}$

Obwohl diese Gruppen der FDJ unterstanden, wurden einige von ihnen schon bald aus unterschiedlichen Gründen wieder aufgelöst. Der IG Jazz Leipzig wurde vorgeworfen, mit „westlichen Agenten“ Verbindung aufgenommen zu haben; nach ihrer Auflösung bereits im Mai

21 Ernst-Ludwig Petrowsky, Beat Blues (Version für Saxophon und Jazztrio Dresden 1965). Ernst-Ludwig Petrowsky (as, ts, ld), Peter Setzmann (p), Uli Türkowsky (e-b), Wolfgang Winkler (dr), CD-Beilage zu Bratfisch: Freie Töne (live aufgenommen am 8./9.12.1965, Kongresssaal im Hygiene-Museum Dresden).

22 Siehe Album Takin' of (1962) mit Herbie Hancock (p), Freddie Hubbard (tr), Dexter Gordon (ts), Butch Warren (b) und Billy Higgins (dr). BLUE NOTE 92759.

23 Bert Noglik, „Hürdenlauf zum freien Spiel. Ein Rückblick auf den Jazz in der DDR“, in: Jazz in Deutschland, hrsg. von Wolfgang Knauer, Hofheim: Wolke 1996 (Darmstädter Beiträge zur Jazzforschung 4), S. 205-221, hier S. 212.

24 Siegfried Schmidt-Joos: Die Stasi swingt nicht, Mitteldeutscher Verlag GmbH, Halle (Saale) S. 11.

25 Ulf Drechsel, Karlheinz Drechsel. Zwischen den Strömungen. Mein Leben mit dem Jaž, Berlin: Jazzwerkstatt 2011, S. 48. 


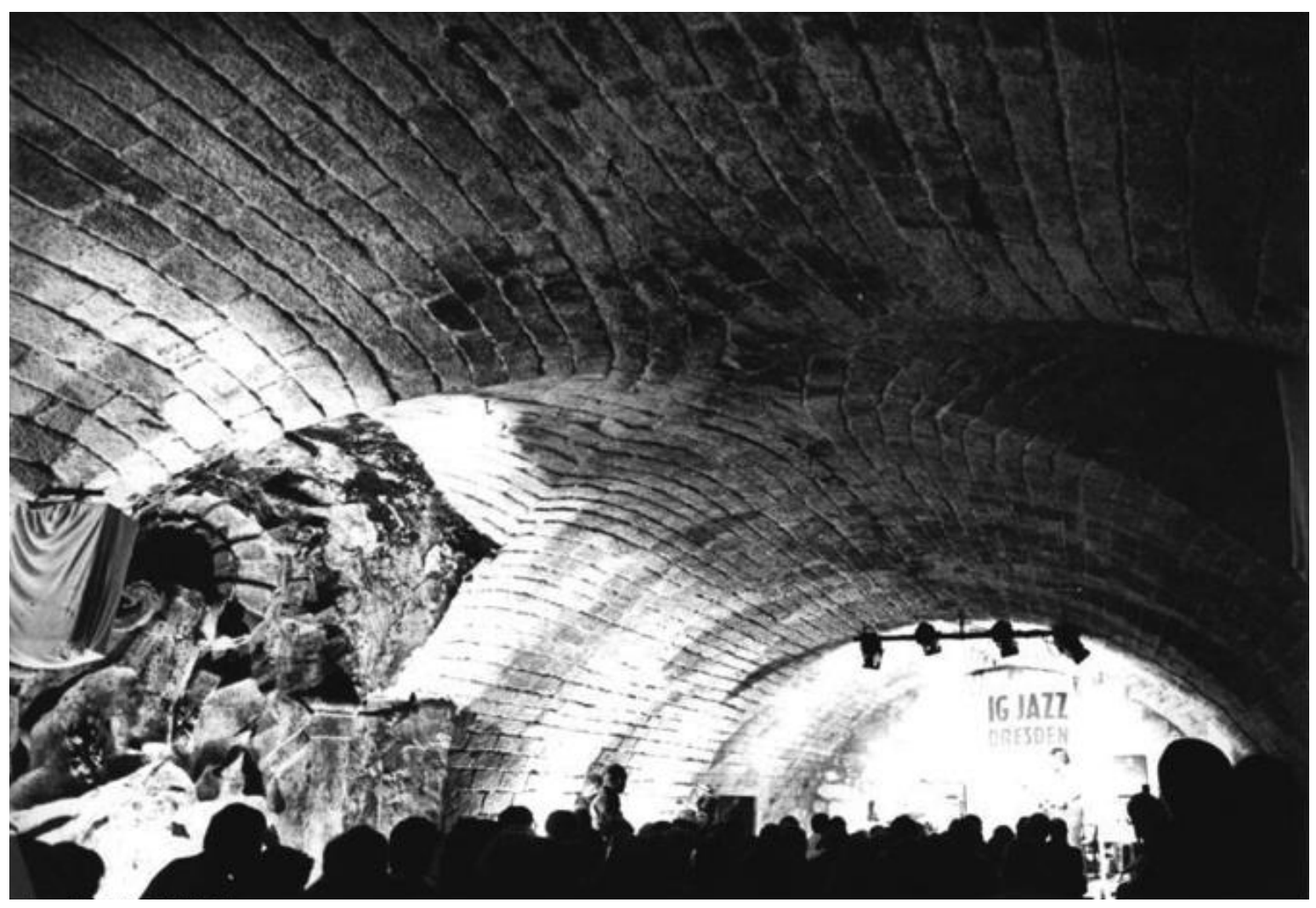

Abbildung 2: IG Jazz Dresden ${ }^{26}$

1955 arbeitete sie im Untergrund weiter. ${ }^{27}$ Der IG Jazz Dresden erging es nicht anders, auch sie wurde bereits ein Jahr nach ihrer Gründung verboten. ${ }^{28} 1977$ kam es zu einer erneuten Gründung dieser IG Jazz innerhalb der Stadtorganisation Dresden und des Kulturbundes der DDR. ${ }^{29}$ Sie arbeitete von da an eng mit dem Dixieland-Festival Dresden zusammen, und im Laufe der Jahre entstand aus der IG Jazz der Jazzclub Tonne Dresden. Die IG Jazz Berlin wurde im Mai 1959 aufgrund eines Beschlusses der Abteilung Kultur des Magistrats aufgelöst. ${ }^{30}$

Im Jahre 1959 wurde in Eisenach der erste Jazzclub der DDR ins Leben gerufen, und mit ihm entstand eine Arbeitsgemeinschaft Jazz, welche bald die erste Jazz-Zeitschrift der DDR,

26 Foto: Ulrich Häßler, 26. November 1981, Quelle: Bundesarchiv, Bild 183-Z1126-301.

27 Der Leiter der IG Jazz Leipzig war Reginald Rudorf, der sich als einer der ersten in der DDR für den Jazz einsetzte und diesen den Funktionären als Musik des Proletariats zu verkaufen versuchte, die der Ideologie des politischen Systems entsprechen würde. Nachdem Reginald Rudorf mehrfach aufgefallen war, wurde er verhaftet und als „amerikanischer Spion“ bezeichnet. Vgl. Reginald Rudorf, Jazz in der Zone, Köln/Berlin: Kiepenheuer 1964.

28 Anhang, „Chronologie des Jazz in der DDR“, in: Bratfisch, Freie Töne, S. 291-311, hier S. 292-293.

29 Ulrich Gumpert erwähnte im Interview mit der Autorin am 1.2.2013, dass sich Jazzclubs dem Kulturbund der DDR anschlossen, „um Ärger vorzubeugen“. Durch den Anschluss ließ man sie gewähren und ,alles verlief reibungslos“.

30 Anhang, „Chronologie des Jazz in der DDR“, in: Bratfisch, Freie Töne, S. 292. 
Regierung der

Deutschen Demokratischen Republik

Ministerium für Staatssicherheit.
Vertrauliche Dienstsache!

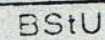

000001

\section{Aktenspiegel}

für die Personalakte des geheimen Informators - Hauptinformators - geheimen Mitarbeiters Inhaber einer konspirativen Wohnung

Reg.-Nr.: $\quad 2505 / 58$

Bestätigt am: $\quad$ 10. 12. 1958

Angeworben am: 10.12. 1958

Angeworben durch: ..... Kienberg/Ruck der bei der

geworbenen Person unter den Namen: Rose/Reich bekannt ist.

Kategorie:

Geheimer Informator

Linie, auf der die geworbene Person arbeitet: Aufkª̈rung von Jazz-ülùos

Abbildung 3: Ausschnitt aus der Akte „Zirkel“ XV 3356/7231

Die Posaune, auf den Markt brachte. ${ }^{32}$ Der SED waren die Interessengruppen und Jazzclubs nicht ganz geheuer, obwohl viele von der FDJ oder vom Kulturbund der DDR betrieben wurden. Um kontrollieren zu können, was sich hinter den Wänden der Jazzclubs und Interessengruppen abspielte, betraute der Staatssicherheitsdienst sogenannte „Geheime Informatoren“ (GI) ${ }^{33}$ mit der Aufgabe, Jazzclubs zu bespitzeln. Sie wurden jedoch nicht nur in der DDR eingesetzt, sondern auch in den Westen gesandt, um Berichte über die dort ansässigen Jazzclubs zu verfassen. Beispielsweise wurde der GI „Zirkel“ für diese Zwecke angeworben, und gleich im Januar 1959 wurden ihm folgende Aufgaben zugeteilt:

„1. Wichtige Veranstaltungen zu besuchen.

2. Dabei feststellen, welche Besucher aus der DDR und dem demokratischen Sektor sind.

3. Wie und in welcher Form wird von $\mathrm{WB}^{34}$ aus Einfluss auf die Jugendlichen der DDR mit welchem Ziel genommen?

31 BStU 1093/91, Seite 1.

32 Noch immer aktiv, wird dieser Klub in der Kulturmälzerei Eisenach betrieben, in der sich auch das Lippmann+Rau-Musikarchiv befindet. Dieses Archiv zählt neben dem Jazzinstitut in Darmstadt zu den größten Jazzsammlungen in Deutschland.

33 Später wurden die Geheimen Informatoren in Inoffizielle Mitarbeiter (IM) umbenannt.

34 Westberlin. 
4. Welche Personen oder Organisationen und Dienststellen nehmen Kontakt zu Jugendlichen aus der DDR auf? ‘35

Diese Aufgabenstellung zeigt die Unsicherheit des Staatsicherheitsdienstes in Bezug auf westliche Einflüsse im Bereich der Tanz- und Unterhaltungsmusik sowie des Jazz.

Auch der Abteilungsleiter des Ministeriums für Kultur ${ }^{36}$ Hans-Georg Uszkoreit wurde im Jahre 1961 als Geheimer Informator angeworben. Er sollte unter dem Decknamen „Amiga“ arbeiten, Dieser GI Amiga war in Bezug auf Musik alles andere als unbedarft; er hatte ein $\mathrm{Mu}$ sikstudium vorzuweisen, war Professor für Musikgeschichte an der Musikhochschule Dresden und ab 1963 Direktor ebenda. Bei seiner Anwerbung wurde er zur aktuellen Situation von Komponisten, Textdichtern und Interpreten befragt; dabei berichtete er von einer ungünstigen Situation auf dem Gebiet der Tanzmusik:

„1. Staatlicherseits haben wir einen vollkommen ungenügenden Überblick über den Einfluss aus

Westdeutschland und Westberlin. Wir haben es nicht in der Hand.

2. Die ideologischen Unsicherheiten und Unklarheiten bei den Autoren und Interpreten.

3. Die Uneinigkeit in unserer Kulturpolitik. “37

Er kritisierte ebenso, dass das Ministerium für Kultur (MfK) gegenüber Rundfunk und Fernsehen nicht weisungsberechtigt sei und so nicht verhindern konnte, dass z. B. ein verbotener Westschlager wie der Kriminaltango von Hazy Osterwald gesendet und dann auch noch dazu getanzt wurde. ${ }^{38}$

Der Jazz war für das System aber „nicht zu fassen“, da er textlos war und „weil sie vom Jazz nichts wussten“, wie es Ulrich Gumpert im Interview begründete. ${ }^{39}$ So wurden bald auch in

35 MFS-AIM 1093/91, S. 22-23.

36 Ursprünglich hieß das Ministerium für Kultur Staatliche Kommission für Kunstangelegenheiten (Stakuko) und wurde mit der Ambition gegründet, das künstlerische Schaffen in der DDR zu fördern und zu lenken. Eines der Anliegen war hierbei der Aufschwung des kulturellen Niveaus der Bevölkerung der DDR. Die Stakuko ging im Jahre 1954 in das Ministerium für Kultur (MfK) über, das in mehrere Abteilungen unterteilt war. Eine der Hauptabteilungen war die HA Darstellende Kunst, Musik und Volkskunst, zu dessen stellvertretender Leitung Hans-Georg Uszkoreit bei deren Gründung 1954 berufen wurde; 1964 wurde er zum Leiter der Hauptabteilung Musik befördert. Uszkoreit war in den 1950er-Jahren bereits Hauptreferent der Stakuko und übernahm mit der Leitung der HA Musik im MfK zugleich die ideologische und organisatorische Lenkung des gesamten Musiklebens in der DDR. In dieser Position war er sogar für mehrere Bereiche der Musik zuständig: Orchesterbesetzungen und Chorwesen, Kontrolle bei Konzertplanungen, Umsetzung der 60:40-Regelung u. a. Uszkoreit, der ab 1963 parallel als Rektor der Musikhochschule Dresden amtete, arbeitete nicht nur an offizieller Stelle für das politische System der DDR, sondern wirkte auch als inoffizieller Mitarbeiter GI Amiga beim Staatssicherheitsdienst mit. Im Jahre 1975 floh er in den Westen.

37 MFS-AIM 5225/77, S. 60-61.

38 Ebd.

39 Interview der Autorin mit Ulrich Gumpert vom 1.2.2013. Ob tatsächlich kein Funktionär Ahnung von Jazz hatte, ist kaum zu beurteilen. Dieser Musikstil schien sich aufgrund seiner Herkunft zunächst nicht mit der Staatsideologie vereinbaren zu lassen und dementsprechend wandten sich die Funktionäre im Auftrag des Staates zuerst einmal gegen die Jazzbewegung. 
der DDR Jazz-Konzertreihen gegründet, wie „Lyrik - Jazz - Prosa“ (1964), „Jazz in der Kammer“ (1965) oder die Jazzwerkstatt Peitz (1973).

\section{Lyrik - Jazz- Prosa}

Im April 1964 wurde in der DDR die Konzertreihe „Jazz und Lyrik“ ins Leben gerufen, bei welcher Jazz in Verbindung mit lyrischen Texten gespielt wurde. Ende 1965 wurde der Name dieser Reihe zu „Lyrik - Jazz - Prosa“ erweitert, ${ }^{40}$ da immer mehr Prosatexte ins Programm aufgenommen wurden. Kulturpolitisch gesehen geschah dies zu einem Zeitpunkt, zu dem es in der DDR liberaler zuging als in den Jahren zuvor: Ab 1963 konnten Westliteratur sowie später als staatskritisch verstandene Bücher auf dem Markt erworben werden. ${ }^{41}$ Unterhaltungsmusik wurde nun gefördert, und auch der Liedermacher Wolf Biermann trat vor überfüllten Auditorien auf. Der Verlag Volk und Welt in Berlin beauftragte den Werbeleiter und Jazzliebhaber Werner „Josh“ Sellhorn mit der Gründung der Konzertreihe „Jazz und Lyrik“, einer Veranstaltungsserie, die Jazz und Lesungen satirischer Gedichte aus den Büchern des Verlags kombinierte, um durch öffentliche Veranstaltungen Reklame für das Verlagsprogramm zu machen. Sellhorn hatte bei der Organisation dieser Veranstaltung einen anderen Hintergedanken als Verlagsleiter Walter Czollek, der ihn mit dieser Aufgabe betraut hatte: Ihm, der wegen seiner Begeisterung für den Bluessänger Josh White den Spitznamen ,Josh“ bekommen hatte, war vom Kulturministerium der DDR untersagt worden, sich öffentlich mit Jazz zu beschäftigen. Dieses Verbot hoffte er nun mit der Konzertreihe unterlaufen zu können. ${ }^{42}$

Sellhorn engagierte die „Jazz Optimisten Berlin“, eine Amateurband, die vorwiegend Dixieland spielte, sowie den Schauspieler und Jazzsänger Manfred Krug für die ersten Veranstaltungen. Das Eröffnungskonzert fand im April 1964 statt und aufgrund des Erfolges kam es in den Jahren 1964 und 1965 zu knapp hundert Aufführungen von „Jazz und Lyrik“ und später „Lyrik - Jazz - Prosa“ in der gesamten Deutschen Demokratischen Republik. Trotz oder vielleicht auch wegen des großen Erfolges hatte das politische Regime ein wachsames Auge auf die Konzertreihe, und sowohl die Auswahl der Texte und Musikstücke als auch die daraus entstandenen Tonträger unterlagen einer Zensur. So benötigte der Mitschnitt eines Konzertes vom Jahre 1965 drei Jahre Wartezeit bis zur Veröffentlichung im Jahre 1968, da u. a. alle Beiträge mit Joachim Kühn herausgeschnitten werden mussten. Dies wurde einerseits mit dem allzu avantgardistischen Klavierspiel von Kühn begründet, vor allem jedoch mit dessen Ausreise 1966. Des Weiteren wurden Passagen wie „Uffjelöst wern wa doch ... rejiert wern wa doch“, das von riesigem Beifall und ,Jawoll“" gefolgt wurde, zensiert. ${ }^{43}$

Am 13. November 1964 trat Wolf Biermann bei „Jazz und Lyrik“ mit vier eigenen Balladen (Die weiße Sophie, Himmelsfahrttag, Mein Vaterland und als Zugabe Deutschland) und zwei

40 Werner Sellhorn, Jazz - Lyrik - Prosa. Zur Geschichte von drei Kultserien, Berlin: Neunplus1 2008, S. 9.

41 Hermann Weber, Die DDR 1945-1990, München: Oldenbourg 52012, S. 67.

42 Sellhorn, Jazz-Lyrik-Prosa, S. 10.

43 Ebd., S. 18-19. 
Chansons von Erich Weinert und Kurt Tucholsky auf, die danach Erwähnung im Bericht des Staatssicherheitsdienstes fanden. Es handelte sich hierbei um Texte, die in den 1920er-Jahren geschrieben worden waren und die sich gegen die „Bonzen“ richteten. Zwei weitere Textteile Biermanns fanden in dem genannten Bericht Erwähnung, nämlich „Wenn ich mal tot bin, werde ich Grenzer und bewache die Grenze zwischen Himmel und Hölle“ und „Wenn ich mal Lust habe, fahre ich in die Hölle und spendiere Stalin ein Bier“. Der Verfasser des Berichtes fand jedoch Genugtuung darin, dass es Biermann seiner Ansicht nach trotz dieser Spitzfindigkeiten nicht gelang, in den Mittelpunkt zu treten. ${ }^{44}$ Auch die Moderation durch Sellhorn blieb nicht ohne Kritik. Er führte durch das Programm und sagte das Weihnachtslied An einen Bonzen ${ }^{45}$ folgendermaßen an: „Es handelt sich um ein Gedicht, das sich sehr kritisch mit den damaligen Verhältnissen auseinandersetzt, aber auch heute noch seine Aktualität besitzt [...]. Natürlich in Westdeutschland. “46 $\mathrm{Ihm}$ wurde der Versuch vorgeworfen, durch die Art seiner Ansage das Publikum zu beeinflussen. Sätze wie „Wir haben uns bemüht, vier brauchbare Balladen herauszusuchen" oder die oben zitierte Ansage waren dem Berichterstatter ein Dorn im Auge, und er betonte in seinem Bericht besonders die lange Pause vor dem Beisatz „Natürlich in Westdeutschland“ und das breite Grinsen von Sellhorn ${ }^{47}$, das der Moderation einen eindeutig sarkastischen Unterton verlieh.

Sellhorn rückte als Jazzveranstalter und vor allem wegen seiner Freundschaft mit Wolf Biermann ins Augenmerk des Systems. Auch blieb es nicht unbemerkt, dass er Wolf Biermann zu einem Konzert von „Lyrik - Jazz - Prosa“ eingeladen und ihm eine Eintrittskarte als Geschenk zugeschickt hatte. Biermann, dem 1965 ein Auftrittsverbot in der DDR auferlegt worden war, kam als Gast zum Konzert von „Lyrik - Jazz - Prosa“ am 13. November 1965. Vor der Kongresshalle wartete jedoch bereits der Staatsicherheitsdienst auf ihn, und Wolf Biermann wurde von der Volkspolizei verhaftet und abtransportiert, was einen Streik des Ensembles auslöste. ${ }^{48}$ Das Publikum harrte ebenso aus und das Konzert begann erst, nachdem ein StasiOffizier mitgeteilt hatte, dass „der Bürger Wolf Biermann soeben wieder auf freien Fuß gesetzt“ worden wäre. ${ }^{49}$

Um die Jahreswende 1966/67 kam es zum vorläufigen Ende von „Lyrik - Jazz Prosa“.${ }^{50}$ Es folgten noch weitere Programme, die Jazz mit anderen Kunstformen verbanden wie „Jazz und Tanz" und eine Reihe mit dem Titel „Jazz \& Folksongs“, bei der wie bereits bei „Lyrik - Jazz - Prosa“ Manfred Krug und die Jazz-Optimisten mitwirkten und Schauspieler wie Eva-

44 BStU, MfS, BV Berlin. AKG 710, S. 4.

45 Die Stasi-Akte verzeichnet den Tucholsky-Titel irrtümlich mit „Über die Bonzen“.

46 BStU, MfS, BV Berlin. AKG 710, S. 3.

47 Ebd., S. 4

48 Sellhorn, Jazz-Lyrik-Prosa, S. 17.

49 Ebd.

50 Ebd., S. 19. 
Maria Hagen und Gerry Wolf sowie das Hootenanny-Quartett ${ }^{51}$ auftraten. Mehrere Plakate für das Konzert vom 4. März 1967 wurden von der FDJ überklebt, um das Konzert so zu verhindern. ${ }^{52}$ Die Konzertreihe wurde 1997 unter dem Namen „Jazz - Lyrik - Prosa“ wiederbelebt; ihre Tradition wird bis heute weitergeführt, auch wenn nicht mehr ausschließlich traditioneller Jazz gespielt wird, sondern hin und wieder auch das Zentralquartett bei den Konzerten mit Texten auftritt. Die Konzertreihe erfreut sich wieder großer Popularität: Auf der Bühne stehen die Jazzmusiker von damals und im Publikum finden sich alle Altersgruppen, großteils aber Fans, die „Lyrik - Jazz - Prosa“ bereits zu DDR-Zeiten erlebt haben und sich nostalgischer Erinnerungen erfreuen.

\section{Ausblick}

„Es steht außer Frage, daß der vergleichsweise hohe instrumentaltechnische und musiktheoretische Standard der Jazzmusiker in der DDR und auch das in Interviews mit ihnen immer wieder durchscheinende hohe musikalische Reflexionsvermögen viel mit diesen institutionellen Gegebenheiten zu tun hat." ${ }^{\text {53 }}$

Wie bereits Ekkehard Jost in seinem Buch Europas Jazz beschrieb, zeichnete sich der Jazz in der DDR durch einen hohen instrumentaltechnischen Standard aus, was daraus resultierte, dass die Musiker eine Berufsausbildung oder ein Studium absolvieren mussten, um eine Spielerlaubnis zu erhalten, die wiederum Bedingung war, um überhaupt Konzerte spielen zu dürfen. Der Jazz in der DDR unterlag der Kontrolle des Systems, aber ein allgemeines Verbot gegen den Jazz wurde nie ausgesprochen. Einzelne Jazzmusiker verließen die DDR, weil sie sich nicht frei genug fühlten, andere arrangierten sich mit dem System, da ihnen die finanzielle Förderung für Kultur zugutekam. Schlussendlich spielten sie sich über die Musik frei.

„Der Zustand der ,Joblosigkeit‘ impliziert nämlich nicht nur persönliche, finanzielle Schwierigkeiten, sondern verhindert zugleich die Bildung von kontinuierlich zusammenwachsenden Ensembles und damit die Entwicklung und Stabilisierung eines gerade für diese von prädeterminierten Schemata unabhängige Musik essentiellen Konzeptes der Gruppenimprovisation.“"54

51 Das Hootenanny-Quartett war eine politische Liedgruppe in der DDR und bestand u. a. aus Uta Schorn, Hans-Georg Pape, Jörn Fechner und Kurt Goldstein. Im Jahre 1967 benannte sich das Quartett nach einer Kampagne der SED gegen Anglizismen in „Oktoberklub“ um.

52 Sellhorn, Jazz-Lyrik-Prosa, S. 20.

53 Jost, Europas Jazz, S. 237.

54 Ekkehard Jost, Free Jaz: Stilkritische Untersuchungen zum Jazz der 1960er Jahre, Hofheim: Wolke Verlag 2002, S. 14. 
Abbildungsnachweise

- Plattencover Zentralquartett (Abb. 1): Idee und Gestaltung: Fritz Magull, Zeichnung: Sigurd Wendland, mit freundlicher Genehmigung (Abb. 1)

- IG Jazz Dresden (Abb. 2): Ulrich Häßler, 26. November 1981, Quelle: Bundesarchiv, Bild 183Z1126-301

- Akte „Zirkel“ XV 3356/72, Seite 1 (Abb. 3): Archiv der Stasi-Unterlagen-Behörde (BStU), Signatur 1093/91. 